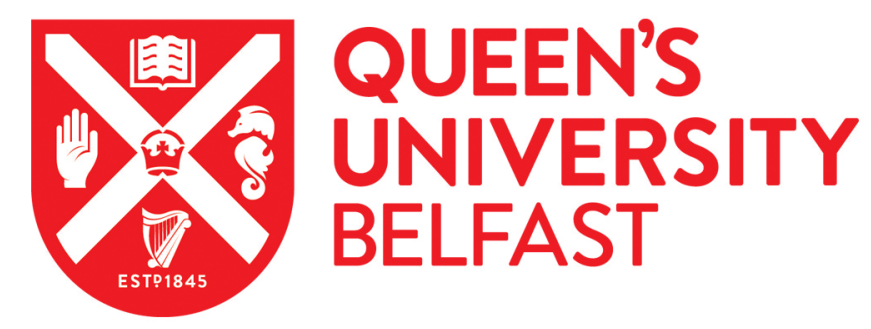

\title{
Extracting the Interfacial Free Energy and Anisotropy from a Smooth Fluctuating Dividing Surface
}

\author{
Baldi, E., Ceriotti, M., \& Tribello, G. A. (2017). Extracting the Interfacial Free Energy and Anisotropy from a \\ Smooth Fluctuating Dividing Surface. Journal of Physics: Condensed Matter. https://doi.org/10.1088/1361- \\ $648 X / a a 893 d$
}

Published in:

Journal of Physics: Condensed Matter

Document Version:

Peer reviewed version

Queen's University Belfast - Research Portal:

Link to publication record in Queen's University Belfast Research Portal

\author{
Publisher rights \\ Copyright 20 the authors. \\ This is an open access article published under a Creative Commons Attribution-NonCommercial-NoDerivs License \\ (https://creativecommons.org/licenses/by-nc-nd/4.0/), which permits distribution and reproduction for non-commercial purposes, provided the \\ author and source are cited.
}

\section{General rights}

Copyright for the publications made accessible via the Queen's University Belfast Research Portal is retained by the author(s) and / or other copyright owners and it is a condition of accessing these publications that users recognise and abide by the legal requirements associated with these rights.

Take down policy

The Research Portal is Queen's institutional repository that provides access to Queen's research output. Every effort has been made to ensure that content in the Research Portal does not infringe any person's rights, or applicable UK laws. If you discover content in the Research Portal that you believe breaches copyright or violates any law, please contact openaccess@qub.ac.uk. 


\title{
Extracting the Interfacial Free Energy and Anisotropy from a Smooth Fluctuating Dividing Surface
}

\author{
Edoardo Baldi ${ }^{1}$ \\ ${ }^{1}$ Laboratory of Computational Science and Modeling \\ Institute of Materials \\ École Polytechnique Fédérale de Lausanne \\ 1015 Lausanne, Switzerland \\ E-mail: edoardo.baldi@epfl.ch \\ Michele Ceriotti $^{1}$ \\ E-mail: michele.ceriotti@epfl.ch
}

\author{
Gareth A. Tribello ${ }^{2}$ \\ ${ }^{2}$ Atomistic Simulation Centre \\ School of Mathematics and Physics \\ Queen's University Belfast \\ Belfast, BT7 1NN, United Kingdom \\ E-mail: gareth.tribello@qub.ac.uk
}

\begin{abstract}
Interfaces between different materials and phases play a crucial role in many physical and chemical phenomena. When performing simulations of matter at the atomic scale, however, it is often not trivial to characterize these interfaces, particularly when they are rough or diffuse. Here we discuss a generalization of a construction, due to Willard and Chandler, that allows one to obtain a smooth dividing surface that follows the irregular, ever changing shape of these fluctuating interfaces. We show how this construction can be used to study the surface that separates a solid material from its melt and how analyses of the Fourier modes for the capillary fluctuations of this instantaneous dividing surface can be performed. This particular analysis is useful as one can compute the specific free energy excess of the interface, and its dependence on orientation relative to the bulk phases, from the average amplitude of the Fourier modes. We therefore discuss the efficiency of this approach, both in terms of system size and statistical sampling.
\end{abstract}

\section{Introduction}

Many interesting phenomena in materials science occur at the interface between regions with different compositions or symmetries [1]. For instance, when a material melts or 
freezes an interface between the solid and liquid phases must form and the creation of this interface introduces a barrier to nucleation [2]. Furthermore, the interface between the liquid and gaseous phases of a material can have chemistry that is different to that in the bulk. These differences can cause solutes to be segregated near to surfaces and even different fractionations or isotopomers near to the surface. [3, 4].

Examining the behavior of the atoms at interfaces using experiments is difficult because in most measurements the signal from the small number of atoms in the vicinity of the interface is washed out by the much stronger signal from the atoms in the bulk. There are of course exceptions to this general rule; namely, experimental techniques that are intrinsically surface-sensitive such as non-linear spectroscopies [5]. These techniques are often difficult to use, however, and there is thus a great deal of interest in using molecular dynamics (MD) simulation to understand the behavior of atoms in the vicinity of interfaces. One problem that can occur when using this technique involves the detection of the interface between the phases or materials. Molecular dynamics, after all, tells one about the motions of all of the individual atoms in the system. It does not tell one about the phases the system is composed of and which atoms together comprise each phase explicitly.

Using the extents of the phases to describe the state of the system is more typical for continuum models such as the phase field method. Data from molecular dynamics trajectories can be used to extract parameters for continuum methods, although it is important to ensure that the connection between the atomistic and the mesoscopic scales is made in a way that is rigorous and transparent [6]. What is required is a robust method for extracting the central quantity in these phase field models, which is the interfacial free energy. There are a number of ways of extracting this quantity from a molecular dynamics simulation of an interface [7-12] but one of the most popular methods involves exploiting Capillary Wave Theory [7, 13]. This method has recently been applied to study model systems such as the hard-sphere fluid [14], and also to realistic models for materials such as metallic nickel [13], sodium chloride [15], and water [16]. In many of these works the position of the interface is determined by examining the average configuration of the atoms within a set of discrete bins. In what follows we discuss an alternative protocol that is based on an elegant solution to the problem of coarse graining an atomistic representation of an interface that was first developed by Willard and Chandler [17]. The fluctuating dividing surface they developed is now regularly used to find the location of the interfaces between a liquid and a vapour phase from a coarse-grained representation of the atomic density [18-20]. Furthermore, this method is perfectly suited to monitoring how the shapes of the phases change as the location of the interface is updated after every MD step. In this paper we discuss an extension of this fluctuating dividing surface construction that can be used to find the interface between bulk phases and discuss how we have implemented this method in the open-source package PLUMED [21]. To demonstrate how this implementation can be employed we then use it to calculate the interfacial free energy between solid and liquid phases of a Lennard-Jones system by means of the Capillary Fluctuation method. 
We show that we are able to extract these quantities from simulations containing much smaller numbers of atoms than have been typically used for these calculations in the past and thus pave the way towards performing these calculations with more computationally expensive interatomic potentials.

\section{Theoretical background}

Willard and Chander's method [17] for finding the interface between phases starts by introducing an instantaneous density field:

$$
\rho(x, y, z)=\sum_{i=1}^{N} K\left(\frac{x-x_{i}}{\lambda}, \frac{y-y_{i}}{\lambda}, \frac{z-z_{i}}{\lambda}\right)
$$

In this expression the sum runs over all the atoms in the system. Each of these atoms is located at a position $\left(x_{i}, y_{i}, z_{i}\right)$ and the $K$ is a smooth, 3-dimensional Kernel function (e.g. a Gaussian) that integrates to one. Each atom thus contributes a function that is peaked at the atom's position and which decays over some characteristic length scale $\lambda$ to the final field $\rho(x, y, z)$.

In a similar spirit, a spatial average of an atom-centered order parameter $\phi_{i}$ provides a smoothly varying field that can be used to distinguish between bulk phases, and that can be regarded as an atomic-scale analogue of a phase field:

$$
\varphi(x, y, z)=\frac{\sum_{i=1}^{N} \phi_{i} K\left(\frac{x-x_{i}}{\lambda}, \frac{y-y_{i}}{\lambda}, \frac{z-z_{i}}{\lambda}\right)}{\sum_{i=1}^{N} K\left(\frac{x-x_{i}}{\lambda}, \frac{y-y_{i}}{\lambda}, \frac{z-z_{i}}{\lambda}\right)}
$$

Here $\phi_{i}$ is some function of the positions of the atoms surrounding the atom $i$ that discriminates between the two phases. Here we focus on order parameters that can be written in the form:

$$
\phi_{i}=\sum_{j \neq i} f\left(\mathbf{r}_{i j}\right) c\left(\left|\mathbf{r}_{i j}\right|\right)
$$

In this expression $\mathbf{r}_{i j}$ is the vector connecting atom $i$ to atom $j$ and $c\left(\left|\mathbf{r}_{i j}\right|\right)$ is a switching function that is equal to one when the length of this vector, $\left|\mathbf{r}_{i j}\right|$, is less than some cutoff and which is zero otherwise. This switching function is used to ensure that the only terms that contribute to the sum are those due to the atoms in the first coordination sphere of atom $i$. The consequence of multiplying by $c\left(\left|\mathbf{r}_{i j}\right|\right)$ is thus that $\phi_{i}$ is related to the average of the function $f\left(\mathbf{r}_{i j}\right)$ calculated over the first coordination sphere. Symmetry functions of this type include the cubic harmonics [10, 12], directional order parameters that measure the relative orientations of molecules [22, 23], the Steinhardt order parameters $[24,25]$ and the local Steinhardt order parameters [25, 26].

Calculating the field, $\varphi(x, y, z)$, using equation 2 is useful when studying the interface between a solid and a liquid phase because the densities of solids and liquids are similar and the density field (equation 1) is thus relatively uniform throughout. Instead it is the symmetry of the structure that changes on moving from the solid to the liquid. 
We therefore use equation 2 to calculate the average value of some symmetry function at each point in the box. Without loss of generality, we will assume the parts of the simulation box where $\varphi$ is large are solid, while the points where $\varphi$ is low are liquid. The interface between these two phases can then be found by finding the 2-dimensional manifold containing points for which:

$$
\varphi(x, y, z)-\varphi_{0}=0
$$

In this expression $\varphi_{0}$ is a parameter and in our PLUMED implementation $\varphi(x, y, z)$ can be calculated using eq. (1) or using eq. (2). This manifold is the Willard-Chandler surface that separates the two phases.

One reason for building this framework for identifying the dividing surface that separates regions of space where the values for a particular atomistic property (i.e. an order parameter) differ is that capillary wave theory (CWT) [27-29] allows one to calculate interfacial properties such as the specific free energy excess of the interface and the interfacial stiffness from the instantaneous local fluctuations of the surface. When the procedure outlined above is looked at in this vein the two-dimensional manifold obtained by solving eq. (3) represents a profile function, $h(\boldsymbol{r})=h(x, y)$, that can be used to quantify the local fluctuations in the position of the interface. According to CWT, the Fourier transform of this profile function - a quantity which is defined via $h(\boldsymbol{r})=\sum_{\boldsymbol{k}} A(\boldsymbol{k}) \exp (i \boldsymbol{k} \cdot \boldsymbol{r})$ - is, in the long-wavelength limit, directly linked to the interfacial stiffness [29]. In particular, the interfacial stiffness and the ensemble average of the long-wavelength Fourier components are related via

$$
\left\langle|A(\boldsymbol{k})|^{2}\right\rangle=\frac{k_{B} T_{m}}{S\left(\widetilde{\gamma}_{11} k_{x}^{2}+\widetilde{\gamma}_{22} k_{y}^{2}+2 \widetilde{\gamma}_{12} k_{x} k_{y}\right)}
$$

Here $k_{B}$ is Boltzmann's constant, $T$ is the temperature, $S$ is the cross-sectional area of the flat interface and the $\widetilde{\gamma}_{i j}$ terms are the elements of the stiffness tensor. The components of this stiffness tensor are equal to second-order coefficients of the power expansion of the interface free energy with respect to orientation $[29,30]$

$$
\widetilde{\gamma}_{i j}=\gamma+\left.\frac{\partial^{2} \gamma}{\partial \theta_{i} \partial \theta_{j}}\right|_{\theta_{i, j}=0}
$$

where, $\widetilde{\gamma}_{i j}(i, j=1,2)$ is the stiffness tensor and $\theta_{i}$ is used to represent an infinitesimal rotation of the interface normal around the $i$ th reference axis in the tangent plane. When defining this tensor an arbitrary coordinate system can be chosen. However, if unit vectors $(\hat{x}, \hat{y})$ oriented along the principal axes of the crystal plane associated with the average interface normal are used, the off-diagonal terms $\widetilde{\gamma}_{i j}(i \neq j)$ are equal to zero.

If relation 4 is used to calculate the stiffness for several independent interfaces, the results can be combined with an analytical expression for the interface free energy $\gamma(\hat{n})$ and the full anisotropy of this function can thus be obtained. Usually the symmetry 
of the system under study determines the kind of expansion used for $\gamma(\hat{n})$. In this work, since we are dealing with Face Centered Cubic crystals, we followed the approach developed by Fehlner [31] that involves cubic harmonics. The expansion used for the interfacial free energy is thus

$$
\gamma(\hat{n}) / \gamma_{0}=1+\varepsilon\left(\sum_{i=1}^{3} n_{i}^{4}-\frac{3}{5}\right)+\delta\left(3 \sum_{i=1}^{3} n_{i}^{4}+66 n_{1}^{2} n_{2}^{2} n_{3}^{2}-\frac{17}{7}\right)+\cdots
$$

where $\hat{n}=\left(n_{1}, n_{2}, n_{3}\right)$ is the interface normal and the parameters $\varepsilon$ and $\delta$ give a measure of the anisotropy. As this relation contains three parameters $\left(\gamma_{0}, \varepsilon\right.$ and $\left.\delta\right)$ at least three independent quantities must be used when fitting the function. In many applications of this Capillary Fluctuation Method in the literature [13, 15, 16, 27, 28] each of these quantities is extracted from a separate simulation of a long and thin simulation cell in which the interface has a "quasi-1D" geometry. In this work, however, the fluctuating interface is a two-dimensional surface that can sustain capillary waves in two directions. As such, if a suitably chosen interface is simulated we can extract two independent stiffness values from a single simulation. As discussed in the next section we can thus extract a fully parametrized $\gamma(\hat{n})$ from simulations of two independent surfaces.

\section{Simulations and results}

The simulations presented in this paper were of an interface between the solid and liquid phases of Lennard-Jones atoms. A truncated form of the Lennard-Jones potential [32] was used throughout and thus the pair potential was given by:

$$
V(r)= \begin{cases}4 \epsilon\left[\left(\frac{\sigma}{r}\right)^{12}-\left(\frac{\sigma}{r}\right)^{6}\right]+C_{1} & r \leq 2.3 \sigma \\ C_{2}\left(\frac{\sigma}{r}\right)^{12}+C_{3}\left(\frac{\sigma}{r}\right)^{6}+C_{4}\left(\frac{\sigma}{r}\right)^{2}+C_{5} & 2.3 \sigma<r<2.5 \sigma \\ 0 & r \geq 2.5 \sigma\end{cases}
$$

where $C_{1}=0.016132 \epsilon, C_{2}=3136.6 \epsilon, C_{3}=-68.069 \epsilon, C_{4}=-0.083312 \epsilon$ and $C_{5}=0.74689 \epsilon$.

All molecular dynamics simulations were performed using LAMMPS at a temperature of 0.6185 Lennard-Jones units. In the simulations the temperature and pressure were kept constant using a Nose-Hoover thermostat and barostat. To monitor which atoms were in the solid parts of the simulation box and which atoms were liquid we used a cubic harmonic symmetry function. This function is further discussed in Appendix A and has been used in a number of other recent works $[10,32,33]$.

To generate an initial configuration containing a solid-liquid interface we took a perfect crystalline FCC unit cell with a lattice parameter that was consistent with the density at the chosen temperature (i.e. the melting temperature $T_{m}=0.6185$ ). As we wanted to simulate solid-liquid interfaces with multiple different orientations we aligned the $z$ axis of the coordinate system for our cell with the normal to the desired surface. We then replicated the unit cell in the $x y$ plane and along the $z$ direction. When doing 
Table 1. Dimensions of the simulations and the number of atoms in each of the cells used in this work. The fourth column gives the cell dimension along the $\hat{z}$ axis, perpendicular to the interface normal. All lengths are given in unit of $\sigma$.

\begin{tabular}{lllll}
\hline Orientation & $L_{x}$ & $L_{y}$ & $L_{z}$ & $N$ \\
\hline$(100)$ & 32.354 & 32.354 & 80.886 & 80000 \\
(110) large & 45.756 & 32.354 & 68.634 & 96000 \\
$(110)$ small & 22.878 & 19.413 & 54.907 & 23040 \\
\hline
\end{tabular}

this we used a large number of replicas in the $z$ direction to ensure that we would have reasonably thick solid and liquid regions. This reduces the severity of finite-size effects and prevents the system from melting or freezing in its entirety.

The initial stages of our molecular dynamics simulations were used to generate the solid-liquid interface. In these early simulations the atoms with $z$ positions in a particular range were held fixed. The size of this fixed region depended on the length of the supercell in the $z$ direction but in general it was set between $L_{z} / 3$ and $2 L_{z} / 3$, where $L_{z}$ is the total length of the box in the $z$-direction. These constraints were kept in place during an MD simulation of approximately 100 LJ time units, with a timestep of 0.004 time units, that was run in $N V T$ ensemble with the temperature fixed well above $T_{m}$. During this simulation the unconstrained portion of the supercell was observed to melt completely so that two solid-liquid interfaces were formed. With these interfaces in place, the system was then equilibrated at the melting temperature, $T_{m}$, for a further 100 time units in the NPT ensemble with the constraints on the atoms in the solid parts of the system still in place. Lastly, the constraints on the solid atoms were removed entirely and a final 100 time units of $N V T$ equilibration of the system were performed. Once this final equilibration had completed a 10'000-time-units, NPT production run was performed.

Interfaces between the (100) and the (110) surfaces and the melt were generated using the procedure outlined in the previous paragraph. For the simulations of the (100) interface a supercell of of $20 \times 20 \times 50$ unit cells along the standard $x, y$ and $z$ crystallographic axes was generated. For the simulations of the (110) interface supercells with the $x, y$ and $z$ axes aligned with the $[\overline{1} 10],[00 \overline{2}]$ and [110] crystallographic directions respectively [29] were generated. There were thus 80000 atoms in our simulations of the (100) interface and and 96000 atoms in the first of our simulations of the (110) interface. In order to assess the validity of our proposed model for fitting the Fourier spectrum we also prepared a cell with a (110) interface that contained roughly a quarter of the atoms that were in this large cell.

We began our analysis by investigating the sensitivity of the procedure outlined in the previous section to the two main parameters in the recipe; namely, the bandwidth of the Gaussian kernel functions in equation 2, $\lambda$, and the spacing between the grid points at which this function is evaluated. These initial tests were performed on trajectories in which the (100) interface was simulated. We found that the ensemble average for the 
Fourier transform of the phase field was almost identical for grid spacings of 0.25 and 0.5 Lennard Jones units and thus opted to use the larger spacing in order to lower the total number of grid points and to thus reduce the computational expense.

Figure 1 shows how the $x$-component of the ensemble average for the Fourier transform changes as the bandwidth, $\lambda$, is adjusted. According to the capillary fluctuation relation $4, A(k)$ should decay following $A(k) \propto 1 / k^{2}$. We find that this rule is followed for small $k$, but that there are clear deviations from this behavior at large $k$. These deviations at large $k$ are unsurprising, however, as the capillary fluctuation dispersion is known to be affected by artifacts that depend on how the interface profile is constructed. These effects are particularly dramatic at wave vectors that describe fluctuations over length-scales of individual atoms but deviations from linearity can also be apparent at much smaller values of $k$ and can thus affect any estimate of the interface stiffness coefficients. When it comes to these deviations there is a clear advantage in using the position of an isocontour of a smooth kernel density to define the position of the interface as we have some indication as to the form these artifacts should take. Eqn. (1) describes the convolution of the atom density with a kernel function - so the Fourier transform of the smoothed density $\rho$ is the point-wise product of the Fourier transform of the density field and the Fourier transform of the kernel function. The onset of the deviations from linearity induced by the smoothing should, therefore, depend on the value of the bandwidth $\lambda$. In particular, as $\lambda$ is increased the onset of the deviation should move to smaller values of $k_{x}$.

To account and correct for the impact of the density smoothing on the capillary fluctuation dispersion, we introduce a different ansatz for eq. (4):

$$
\left\langle|A(\boldsymbol{k})|^{2}\right\rangle=\frac{k_{B} T \exp \left[-k_{x}^{2} / 2 \xi_{x}^{2}-k_{y}^{2} / 2 \xi_{y}^{2}\right]}{S\left(k_{x}^{2} \widetilde{\gamma}_{11}+k_{y}^{2} \widetilde{\gamma}_{22}+2 k_{x} k_{y} \widetilde{\gamma}_{12}\right)},
$$

This is the form that would result from convoluting the "ideal" interface profile with a Gaussian function. This argument is a slight oversimplification of the matter, however, as the expression for the phase field eq. (2) is not just a convolution of the atomic order parameters. We are, after all, computing an isocontour of the smoothed field and are not smoothing the isocontour. For this reason, we thus treated the smoothing bandwidths $\xi_{x}$ and $\xi_{y}$ as fitting parameters. We find however that the best fit values typically differ by less than $25 \%$ from the value of $1 / \lambda$ that would be expected if the Gaussian convolution argument could be taken at face value so our ansatz is justified retrospectively. Better still, we observe that when the data is modelled using equation 8 rather than equation 4 , and when $\xi_{x}$ and $\xi_{y}$ are used as fitting parameters, the artifacts due to the smoothing are taken into account and the final values obtained for $\widetilde{\gamma}_{11}, \widetilde{\gamma}_{22}$ and $\widetilde{\gamma}_{12}$ do not depend significantly on the value used for the bandwidth $\lambda$, and the range of values for $k$ that are included in the fit. In other words, the values obtained from our method end up being relatively insensitive to the hyper-parameter $\lambda$.

The fact that, in comparison to an analysis based on (4), an analysis based on (8) is insensitive to manner in which the dividing surface is constructed, leads to significant 


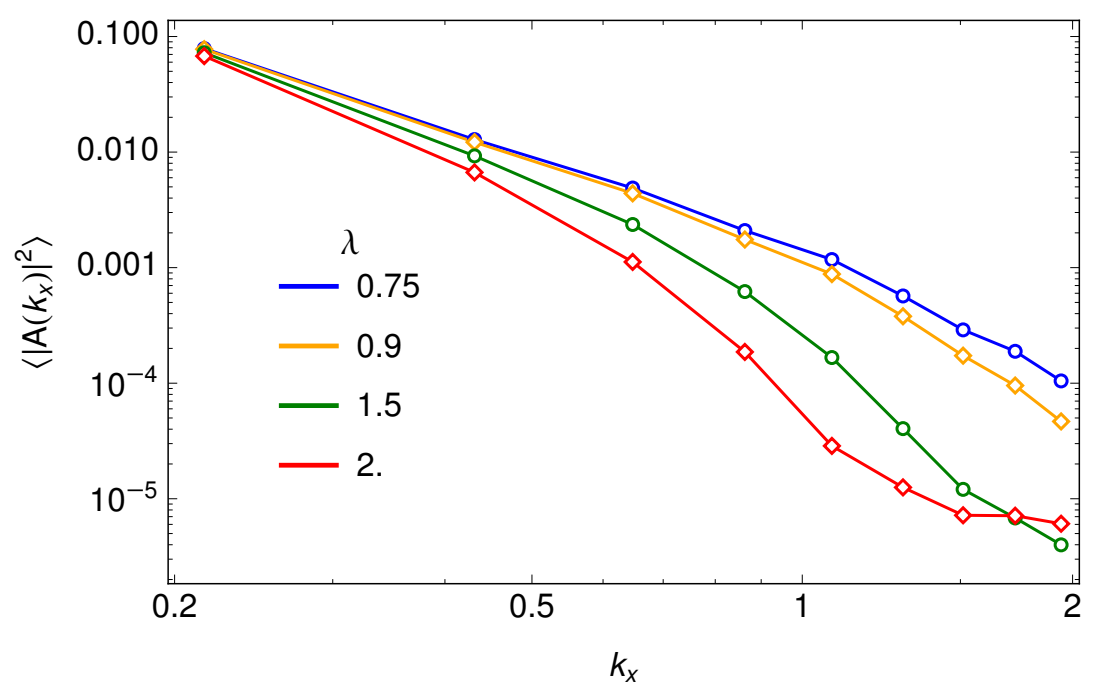

Figure 1. Fitted Fourier spectra along the $k_{x}$-direction for a range of $\lambda$ values. The $\lambda$ parameter controls the extents of the kernel functions that are used when constructing the coarse grained field in eq. (2).

computational advantages. For instance, as discussed in the previous paragraph, Eqn. (4) is only valid in the macroscopic limit as the amplitude of short-wavelength (large $k$ ) fluctuations depends on the manner in which the height profile is constructed. In previous simulation works on CFM very large simulation cells have thus been employed and $\left\langle\left|A\left(k_{x}, k_{y}\right)\right|^{2}\right\rangle$ has been calculated for as many small $k$ values as possible. The fact that we can correct for the artifacts induced by the smoothing of the phase field ensures that we, by contrast, are able to extract meaningful information from larger values of $k$, and thus allows us to reduce the size of the simulation. Figures 2 and 3 show that we can thus get values for the stiffness that are consistent with those in previous works even though we use a much smaller simulation cell, and that we can extend the fitting to large values of $k$, as long as we use eq. (8) in place of eq. (4) when performing the fitting. In fact figs. 2 and 3 show the values of the stiffness coefficients that we extract as a function of the radius of the region in $k$-space, $k_{\max }$, from which we take data. Obviously, when this radius is made larger we include more data points in the fitting, thereby reducing statistical uncertainety.

A further benefit of using the information on $\left\langle\left|A\left(k_{x}, k_{y}\right)\right|^{2}\right\rangle$ for large values of $k_{x}$ and $k_{y}$ is that it allows us to reduce the amount of time for which simulations have to be run to achieve statistical convergence. The dynamics of $A\left(k_{x}, k_{y}\right)$ can be seen as that of a harmonic oscillator of frequency $\omega \propto k$ undergoing overdamped Langevin dynamics. The autocorrelation time for such processes is proportional to $1 / \omega^{2}$ (see e.g. Ref. [34]) so longer simulations are required to converge the averages associated with the modes with long wavelengths. Furthermore, difficulties associated with converging the Fourier amplitude fluctuations for the longer wavelength modes might also be the cause of the non-Gaussian behavior that was observed for these modes in simulations of the interface between solid and liquid sodium chloride [15]. Figure 5 shows that this analysis accords 


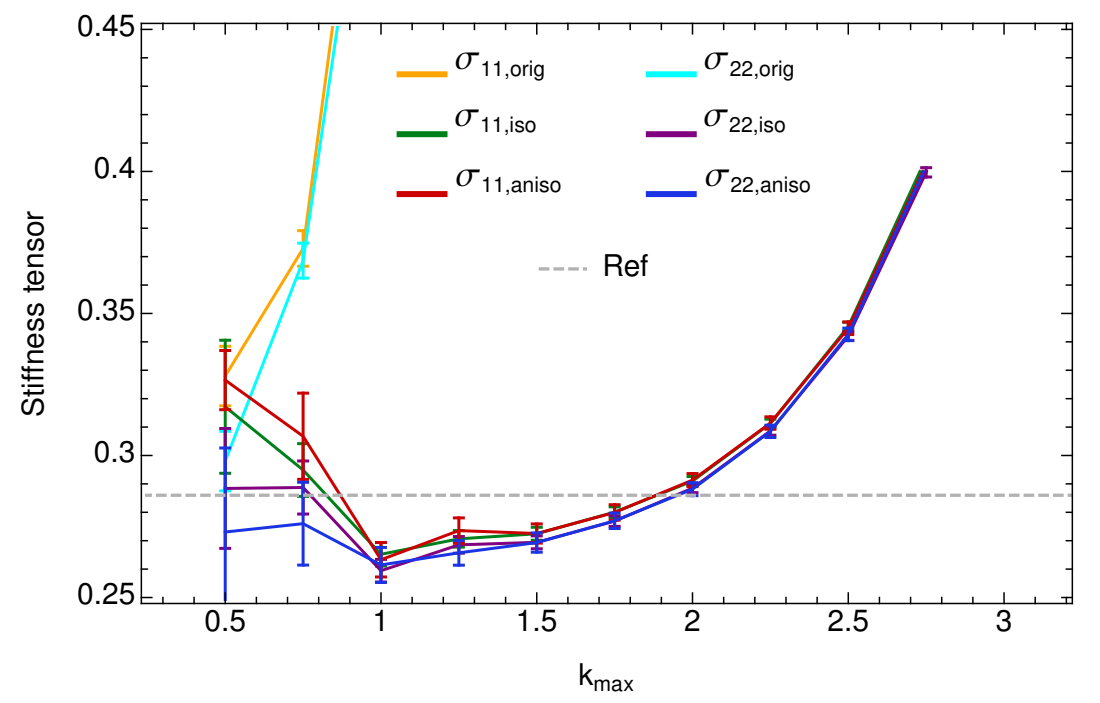

Figure 2. Fitted values for the stiffness tensor of the (100) interface as a function of the radius of the cutoff in $k$-space. The points on the lines labelled orig were obtained by performing fits using eq. (4), which is the model that has been widely used in other works on CFM. The points on the line labelled aniso, meanwhile, were calculated by fitting using eq. (8) and the points on the line labelled iso were fitted using a modified, isotropic version of eq. (8) in which $\xi_{x}=\xi_{y}$. The horizontal, grey dashed line indicates the value of the stiffness that was found in reference [29]. It is clear from this figure that, when the fit is performed using eq. (8), stiffness values that are consistent with the known values of this quantity can be obtained for a range of $k_{\max }$ values. By contrast, when the fit is performed using eq. (4) the value of the stiffness differs substantially from the known values for all but the smallest values of $k_{\max }$. This is unfortunate as results in the region close to $k=0$ are affected by substantial errors as only a small number of points are included in the fit. For this interface the anisotropic and isotropic versions of eq. (4) give very similar values for the stiffness tensor. This makes sense though as the (100) interface only has one independent value for the stiffness tensor.

with the observations we make for our system. We find that the autocorrelation time for the modes increases with $1 / k^{2}$ when $k$ is small. In conclusion, the fact that we are able to use information on $\left\langle\left|A\left(k_{x}, k_{y}\right)\right|^{2}\right\rangle$ at relatively large values of $k_{x}$ and $k_{y}$ when fitting using eq. (8) ensures that we can extract information from relatively short-timescale simulations run with relatively few particles.

Figure 3 illustrates why we use an anisotropic kernel in eq. (8). Our experience here is that for an isotropic surface such as the (100) the inclusion of the additional parameter makes very little difference. We get very similar values for $\widetilde{\gamma}_{11}$ and $\widetilde{\gamma}_{22}$ if we use an isotropic kernel and enforce $\xi_{x}$ and $\xi_{y}$ to be equal in the fit. However, for an anisotropic surface such as the (110) we see weaker dependence on $k_{\max }$ and much closer agreement between our results and the results in the literature when we include separate $\xi_{x}$ and $\xi_{y}$ parameters in the fit. Furthermore, the fitted values for $\widetilde{\gamma}_{11}$ and $\widetilde{\gamma}_{22}$ are more consistent across the range of $k_{\max }$ values when the $\xi_{x}$ and $\xi_{y}$ parameters are allowed to vary independently during the fit.

The values of the stiffness presented in fig. 3 were obtained by analyzing the smaller 


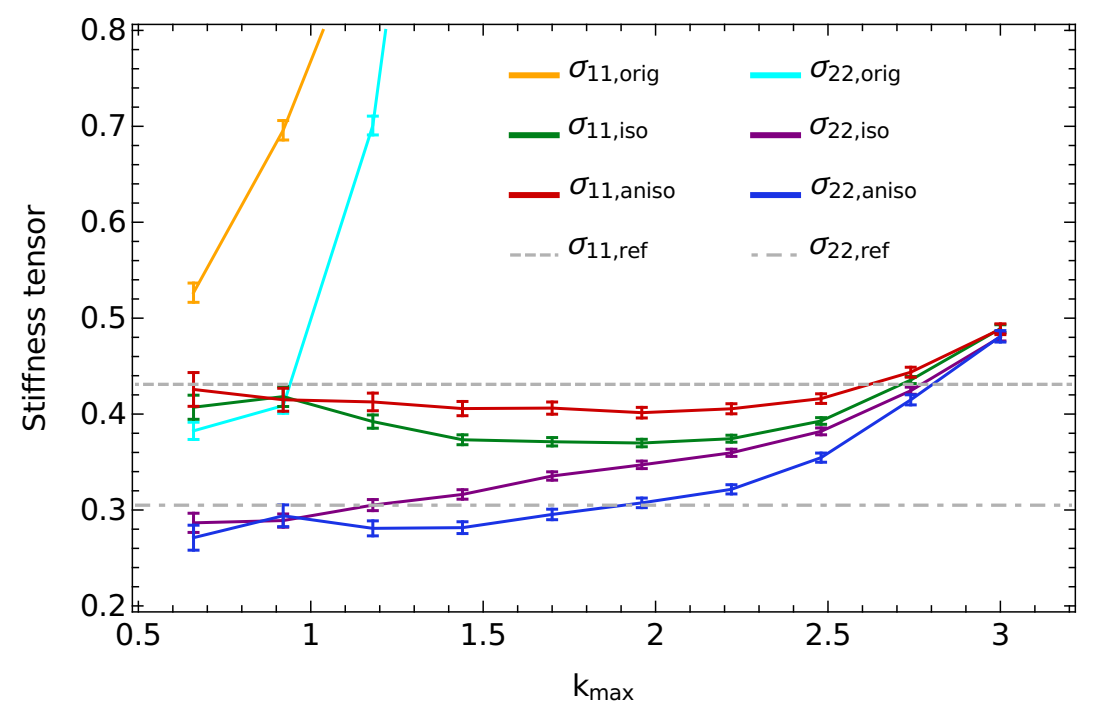

Figure 3. Fitted values for the stiffness tensor of the (110) interface as a function of the radius of the cutoff in $k$-space. The labels used in legend that describe each of the lines are explained in the caption to fig. 2. This figure once again demonstrates that fitting using eq. (4) only gives values for the stiffness that are consistent with the literature values when $k_{\max }$ is set equal to a small value. If, however, the data is fit using eq. (8) values for the elements of the stiffness tensor that are consistent with the known literature values of these quantities are obtained for a range of values of $k_{\max }$. It is interesting to note that the (110) interface, unlike the (100) interface, has two independent values in its stiffness tensor. It would seem that fitting using the anisotropic version of eq. (8) gives values for the stiffness tensor that agree most closely with those from Becker et al. [29]. When the isotropic version of this model is used the values obtained for the stiffness tensor are pulled closer together than they should be.

of the two simulations that were run on this particular interface. Figure 4 shows the values for the stiffness that were obtained from this simulation together with the values that were obtained by analyzing the larger of the two simulation cells. The size of the cell in this larger simulation is comparable with the size used in the work by Becker and coworkers [29] from which we took the values we have used as a reference. Figure 4 makes clear that, as discussed in previous paragraphs, we can indeed use much smaller simulation cells when we fit using eq. (8) in place of eq. (4). As the figure shows the values for the elements of the stiffness tensor that we obtain from these smaller simulations are within $0.02\left(\epsilon / \sigma^{2}\right)$ of the reference values and are in some cases closer to these reference values than the values we obtain from the larger simulations.

The final stiffness values that we obtain are reported in table 2 together with error bars that were calculated using block averaging $[35,36]$ with a block length that was determined based on the auto-correlation times for the $\left(2 \pi j_{x} / L_{x}, 0\right)$ and $\left(0,2 \pi j_{y} / L_{y}\right)$ components of the Fourier transform $A\left(k_{x}, k_{y}\right)$. The values that we obtain for the stiffness values are consistent with those from the the previous work of Becker and coworkers [29]. However, as discussed in the previous paragraphs, we were able to use 


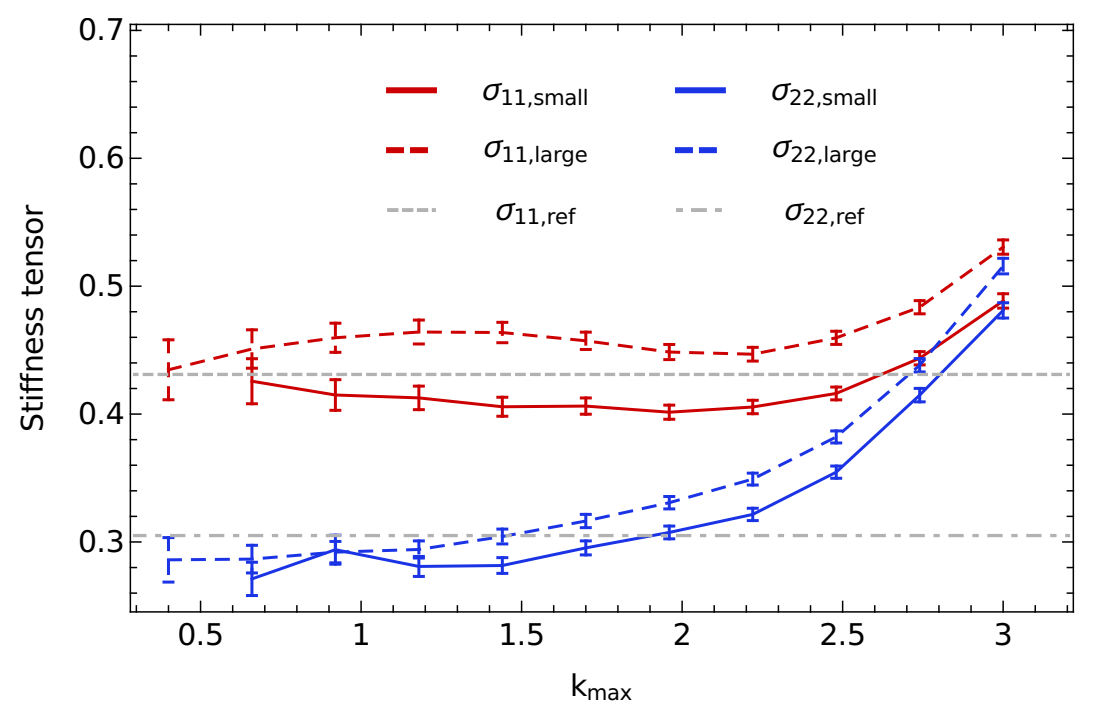

Figure 4. Fitted values for the stiffness tensor of the (110) interface as a function of the radius of the cutoff in $k$-space. All the values on this figure were obtained by performing fits using eq. (8). The dashed lines give the values that were obtained from simulation of 96000 atoms, while the solid lines are the values obtained from simulation cells containing only 24000 atoms.

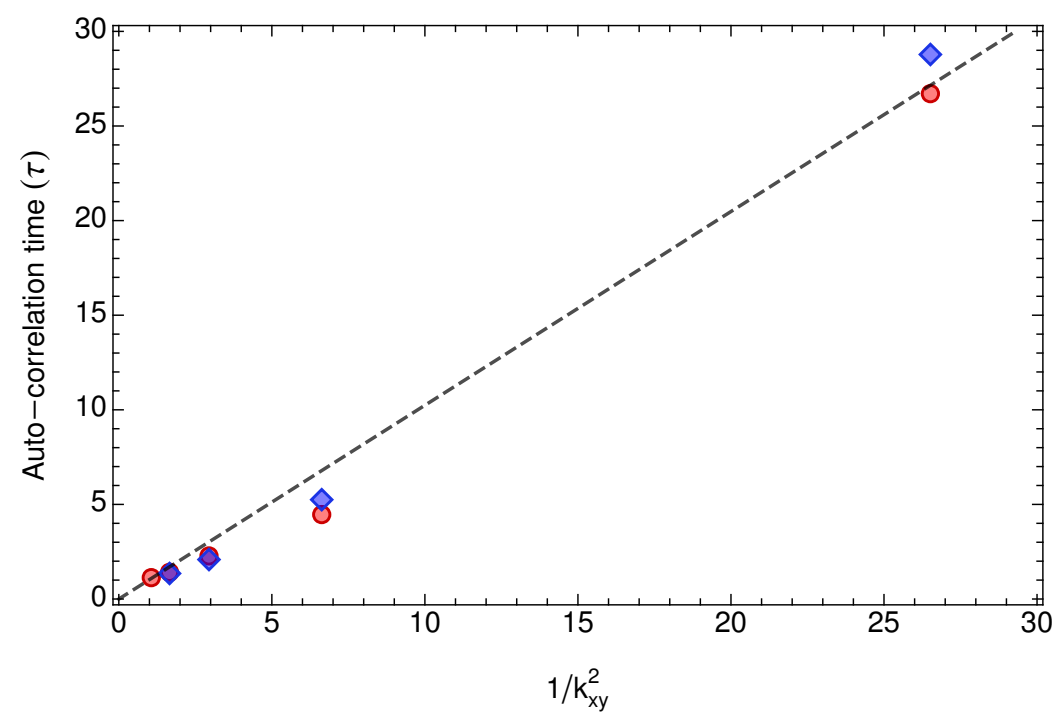

Figure 5. Plot illustrating the auto-correlation time, $\tau$, for $A\left(k_{x}, k_{y}\right)$ in Lennard-Jones reduced units of $\sqrt{m \sigma^{2} / \epsilon}$ as a function of $k_{x}$ (red circles) and $k_{y}$ (blue diamonds), for the (100) interface.

much smaller simulation cells because we use a fluctuating dividing surface construction and because we perform the fit using an expression (eq. (8)) that models the short wavelength modes of the dividing surface using a Gaussian convolution.

We can use the values we have obtained for the stiffness to obtain information on the interface free energy $\gamma(\hat{n})$ by using this data to find parameters for eq. (6). The 
Table 2. Stiffness values for the (100) and (110) interfaces calculated using our method at a reduced temperature of 0.6185 . The values of the stiffness are given in units of $\left(\epsilon / \sigma^{2}\right)$ where $\epsilon$ and $\sigma$ are the values of the Lennard-Jones parameters. The values obtained by Becker and coworkers [29] at the same temperature are also reported for comparison. The error bars for our values represent the $95 \%$ confidence level.

\begin{tabular}{lllll}
\hline & \multicolumn{3}{c}{$\widetilde{\gamma}_{11}$} & \multicolumn{3}{c}{$\widetilde{\gamma}_{22}$} \\
\cline { 2 - 5 } Orientation & Our work & Ref. & Our work & Ref. \\
\hline$(100)$ & $0.2897 \pm 8.0 \times 10^{-4}$ & 0.2866 & $0.2871 \pm 7.0 \times 10^{-4}$ & $=\widetilde{\gamma}_{11}$ \\
$(110)$ & $0.429 \pm 4.0 \times 10^{-3}$ & 0.431 & $0.271 \pm 3.0 \times 10^{-3}$ & 0.305 \\
\hline
\end{tabular}

Table 3. Expressions for the interface free energy and the stiffness derived from eqs. (5) and (6) for different orientations of the interface. The first column contains the Miller indices of the interface together with one of the two directions we used in the tangent plane when determining the stiffness tensor.

\begin{tabular}{llll}
\hline Direction & $\gamma / \gamma_{0}$ & $\tilde{\gamma} / \gamma_{0}$ \\
\hline (100) [010] & $1+\frac{2}{5} \varepsilon+\frac{4}{7} \delta$ & $1-\frac{18}{5} \varepsilon-\frac{80}{7} \delta$ & \\
$(110)[1 \overline{1} 0]$ & $1+\frac{7}{5} \varepsilon+\frac{25}{7} \delta$ & $\left(\begin{array}{cc}1-\frac{21}{10} \varepsilon+\frac{365}{14} \delta & 0 \\
0 & 1+\frac{39}{10} \varepsilon+\frac{155}{14} \delta\end{array}\right)$ \\
$(111)[\overline{1} 10]$ & $1+\frac{12}{5} \varepsilon+\frac{508}{7} \delta$ & $1+\frac{12}{5} \varepsilon-\frac{1280}{63} \delta$ & \\
\hline
\end{tabular}

first step in doing so is to substitute the components of the interface normal vector into eq. (6) and to thus derive analytic expressions for the interfacial free energy, $\gamma(\hat{n})$, for each interface. The first column in table 3 contains the resulting expressions for the (100), the (110) and (111) interfaces. Analytic expressions for the components of the stiffness tensor, $\widetilde{\gamma}_{i j}$, can be derived from these expressions by exploiting eq. (5). These expressions can be found in the second column of table 3.

We have extracted three values for the components of the stiffness tensors, $\widetilde{\gamma}$, of the (100) and (110) surfaces by analyzing the Fourier components of the capillary fluctuations in our MD simulations. We can thus extract the parameters of eq. (6) by solving the three simultaneous equations for these quantities in the last column and the first two rows of table 3. When we do so we find that $\gamma_{0}=0.3558(0.355) \epsilon / \sigma^{2}$, $\varepsilon=0.064(0.057)$ and $\delta=-0.0039(-0.0009)$. When these values are plugged into eq. (6) we obtain values for the interfacial free energy along high-symmetry directions. These values are reported in table 4 where they are also compared with the values obtained using CFM in Ref. [29] and the values obtained using metadynamics [37] in Ref. [38]. Despite the noticeable discrepancies in the values of the stiffness, the fitted values of $\gamma$ are in near-perfect agreement with previous results based on CFM calculations. There is a more significant discrepancy between the values we obtained and the values that were not calculated by performing a fit based on stiffness but which were instead computed directly using an accelerated free energy method. Even so it is remarkable that all these 
Table 4. Specific free energy for the solid-liquid interface in a LJ system at the melting temperature, computed along high-symmetry directions. The present results are compared with those in the previous works of Becker [29] and Cheng [38]. The free energy is in units of $\epsilon / \sigma^{2}$. The figure on the right represents the variation of the surface free energy $\gamma$ as a function of interfacial orientation.

\begin{tabular}{lccc}
\hline$\gamma_{h k l}$ & $\gamma_{100}$ & $\gamma_{110}$ & $\gamma_{111}$ \\
\hline Our work & 0.364 & 0.355 & 0.348 \\
Becker et al. & 0.363 & 0.354 & 0.350 \\
Cheng et al. & 0.373 & 0.360 & 0.352 \\
\hline
\end{tabular}

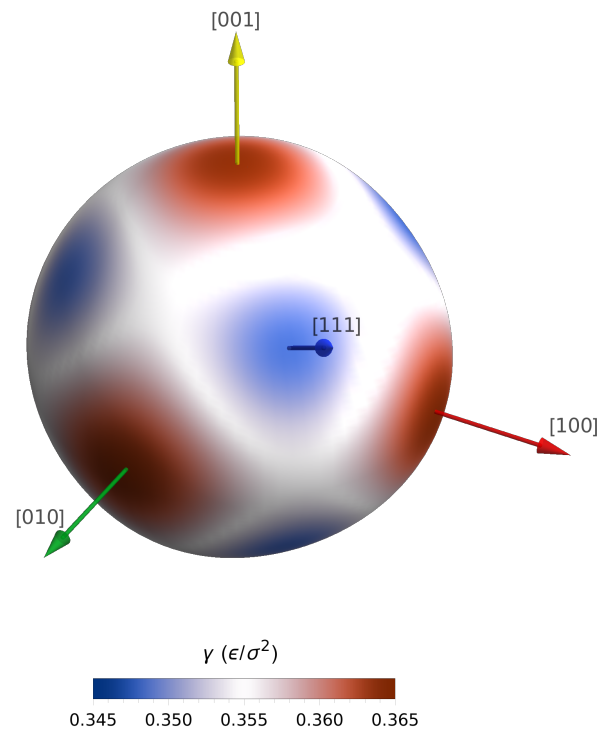

Figure 6. Graphical representation that illustrates the dependence of the interface free energy on the orientation of the interface. The high symmetry directions are highlighted and it is clear that the (100) surface has a higher interface free energy than the (111) surface.

methods can capture the anisotropy between different directions, given the difficulties associated with measuring and computing solid-liquid interfacial properties and also given that the Lennard-Jones potential is known to exhibit a very isotropic interfacial free energy. Being able to compute this quantity is critical as small anisotropies underlay important phenomena such as the formation of dendrites during the solidification of metals and alloys.

\section{Conclusion}

In the preceding sections we have discussed the development of a framework for finding the location of the interface between two phases that differ either because they have a different molar volume or a different local symmetry. This framework extends the Willard-Chandler construction for the liquid-vapor fluctuating interface and takes the positions of all the atoms in the system as input. It returns a two dimensional profile that gives the height of the interface as a function of the two coordinates in the plane perpendicular to the surface normal. We showed how we can examine local fluctuations in the height of this interface using the tools of capillary wave theory and thus extract information on surface properties such as interfacial stiffness and the interfacial free 
energy. The details of the procedure, and the implementation in the open source package PLUMED [21] are discussed in more detail in the appendices.

We have shown that defining the height profile of the interface using a smooth "phase field" offers significant advantages in terms of computational efficiency when it comes to determining interface free-energies and anisotropies using the capillaryfluctuation method. In particular, the form of the field suggests that a Gaussian convolution ansatz should be used to describe the deviation of $\left\langle\left|A\left(k_{x}, k_{y}\right)\right|^{2}\right\rangle$ from the asymptotic $1 / k^{2}$ behavior that occurs at short wavelengths. This ansatz makes it possible to extract meaningful information from larger values of the wavevector. Consequently, a reasonable number of usable Fourier components can be extracted from a relatively-small simulation box so the computational cost of the CFM method can thus be reduced. What is more, since shorter-wavelength fluctuations have smaller autocorrelation times, it is also possible to use shorter simulation times.

The fact that we can calculate stiffness values from shorter simulations containing smaller number of atoms combined with the availability of a robust implementation of this technique in a widely-used simulation package ensures that we are now in a position to apply these powerful techniques when investigating more complicated and more realistic interatomic potentials. Furthermore, the fact that we can investigate the fluctuating dividing surface between (meta)stable phases, using an arbitrary order parameter that is capable of distinguishing between their local atomic environments opens up new opportunities for studying the structure and thermodynamics of interfaces.

\section{Acknoledgments}

The authors gratefully acknowledge financial support from the CCMX Project " $\mathrm{AM}^{3}$ ", including the industrial partners involved: Asulab, Audemars Piguet, General Electrics, Heraeus, Oerlikon Metco, Patek-Philippe, Rolex, Varinor.

\section{Appendix A. An order parameter for FCC symmetry}

The symmetry function that was used in this work to distinguish atoms in the solid part of the simulation box from those in the liquid part has been already employed in a number of other recent works $[12,33]$. It was specifically designed to identify atomic environments in which the orientation of nearest neighbors is consistent with $f c c$ packing and has the form shown below:

$$
s_{i}=\frac{\sum_{j \neq i} \rho\left(\mathbf{R} \cdot \mathbf{r}_{i j}\right) \Theta\left(\left|\mathbf{r}_{i j}\right|\right)}{\sum_{j \neq i} \Theta\left(\left|\mathbf{r}_{i j}\right|\right)},
$$

$$
\text { where } \rho\left(\mathbf{R} \cdot \mathbf{r}_{i j}\right)=\frac{x^{4} y^{4}+x^{4} z^{4}+y^{4} z^{4}}{\left|\mathbf{r}_{i j}\right|^{8}}-\frac{\alpha x^{4} y^{4} z^{4}}{\left|\mathbf{r}_{i j}\right|^{12}} \text {. }
$$

In these expressions $\alpha$ is a parameter that is set equal to 27 , which is the value that allows the function to best resolve between solid and liquid configurations. $\mathbf{r}_{i j}$ is the 
vector connecting the central atom $i$ to atom $j$ and $\Theta$ is a switching function that acts on the length of the vector $\mathbf{r}_{i j}$. This function has the following form:

$$
\Theta(x)= \begin{cases}1 & x \leq d_{0} \\ 0 & x \geq d_{1} \\ (y-1)^{2}(1+2 y) & d_{0}<x<d_{1} \quad \text { where } y=\frac{x-d_{0}}{d_{1}-d_{0}}\end{cases}
$$

with parameters $d_{0}=1.2$ and $d_{1}=1.5$. In equation A.1 the matrix $\mathbf{R}$ is used to rotate the vector $\mathbf{r}_{i j}$. The symbols $x, y$ and $z$ are then used to represent the elements of the rotated vector $\mathbf{R} \cdot \mathbf{r}_{i j}$. It is possible to show that $s_{i}=1 / 16$ when the atoms in the first coordination sphere around atom $i$ are at their ideal $f c c$ lattice sites. This is only true, however, if the simulation cell vectors are aligned with the unit cell vectors for the $f c c$ structure. Obviously, if we are investigating the properties of the (110) surface, this will not be the case as the simulation cell will have been set up so that the (110) direction runs parallel to the $z$ direction so as to ensure that the (110) surface forms in the $x y$-plane. The rotation matrix that appears in the definition of the symmetry function thus ensures that atoms with neighbors arranged as they would be in an ideal $f c c$ structure with the desired orientation have an $s_{i}$ value equal to $1 / 16$.

If the distribution of $\mathbf{r}_{i j}$ values around the central atom $i$ is uniform, as it would be in a liquid, then $s_{i}=\frac{143-\alpha}{5005}$. We thus use this value and the value of $s_{i}$ for an ideal $f c c$ crystal to linearly scale the $s_{i}$ values calculated using eq. (A.1) to a value between 0 and 1. Distributions for the values of the resulting quantities for the atoms in a bulk solid and a bulk liquid are shown in fig. A1. This figure shows that there is very little overlap between the distribution of values in bulk solid and bulk liquid. To make these distributions even more strongly peaked and the distinction between atoms in the liquid and solid parts even clearer, however, we transform the linearly-scaled $s_{i}$ values by the switching function shown in fig. A1, which has the functional form shown below:

$$
S(x)=1-\left[1+\left(2^{a / b}-1\right)\left(\frac{x}{r_{0}}\right)^{a}\right]^{-b / a}
$$

with parameters $a=8, b=8$ and $r_{0}=0.45$. This procedure ensures that any atoms that sit in an environment that is similar to that of the solid have a symmetry function value close to one and that atoms in the liquid have a symmetry function value of close to zero.

\section{Appendix B. Implementation}

In the following sections, we discuss the implementation of the fluctuating dividing surface method in PLUMED in detail and present a commented input file for a CFM simulation. 


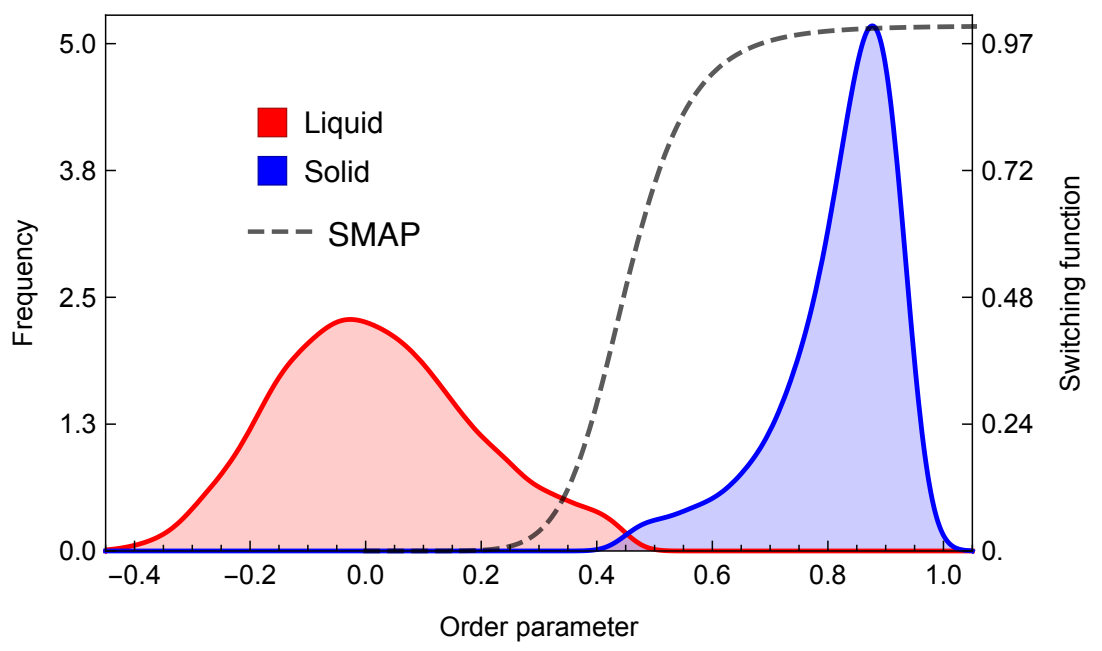

Figure A1. Probability density distribution functions for the order parameter that is described in this section. The histograms shown in red and blue in this figure were calculated from a snapshot from the (110) simulation. The dashed line shows the switching function, eq. (A.2), that was used to convert the order parameter values in order to have a quantity that better distinguished the solid from the liquid.

Appendix B.1. Density profiles

A method that has been frequently used to analyse simulations in which there is an interface between two materials involves calculating the ensemble average for either the density or the the weighted average of some symmetry function as a function of $z$ - the direction perpendicular to the interface. An input similar to the one shown below allows us to calculate one such profile using PLUMED.

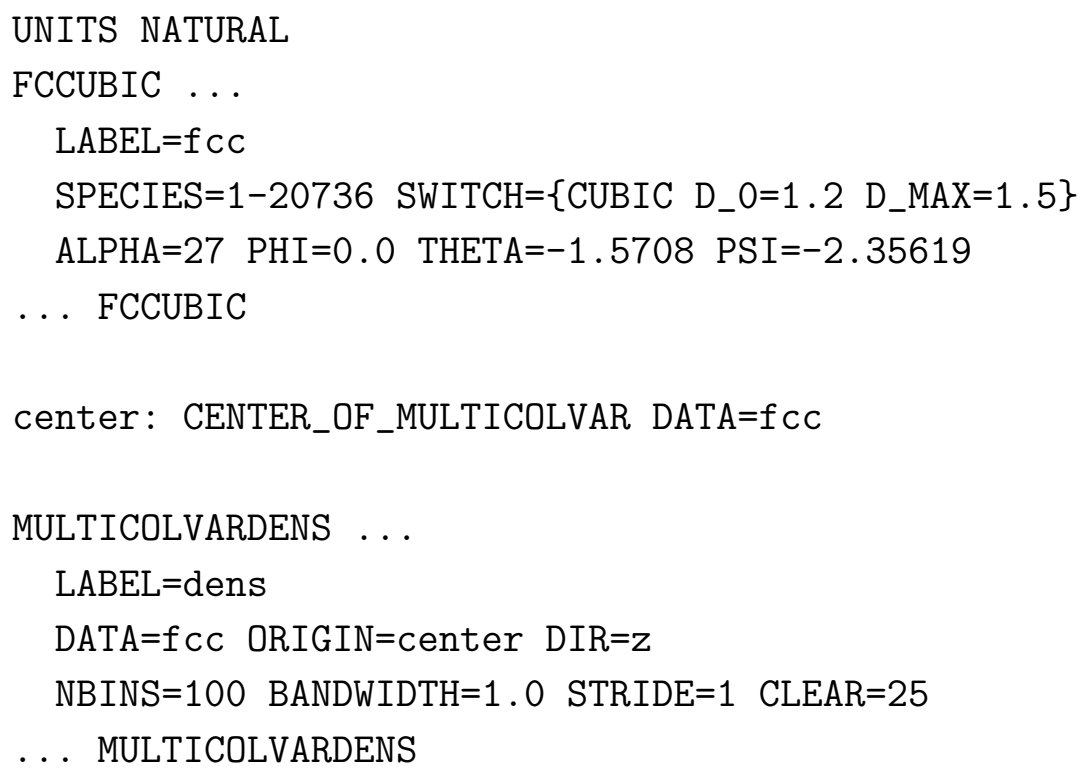

This input tells PLUMED that we would like to calculate the scaled $s_{i}$ parameter 
introduced in equation A.1 for all of the $N$ atoms in the system. These $s_{i}$ values are then inserted into the equation below:

$$
\langle\varphi(z)\rangle=\left\langle\frac{\sum_{i=1}^{N} s_{i} K\left(\frac{z-z_{i}}{\lambda}\right)}{\sum_{i=1}^{N} K\left(\frac{z-z_{i}}{\lambda}\right)}\right\rangle
$$

In this expression $K$ is a normalized kernel function (in this work a Gaussian with bandwidth set equal to $1.0 \sigma$, where $\sigma$ stands for the unit of length in Lennard-Jones reduced scheme) and $z_{i}$ is the $z$ coordinate of the $i$ th atom relative to a reference position. In this work this reference position is located in the centre of the solid region of the system and is found by taking the following weighted sum:

$$
z_{\mathrm{ref}}=\frac{L_{z}}{2 \pi} \tan ^{-1}\left(\frac{\sum_{i=1}^{N} s_{i} \sin \left(\frac{2 \pi z_{i}}{L_{z}}\right)}{\sum_{i=1}^{N} s_{i} \cos \left(\frac{2 \pi z_{i}}{L_{z}}\right)}\right)
$$

In this expression $L_{z}$ is the length of the simulation cell in the $z$ direction, $s_{i}$ is the value of eq. (A.1) evaluated from the positions of the atoms around atom $i$ and $z_{i}$ is the $z$ coordinate of atom $i$. This quantity is calculated by the CENTER_OF_MULTICOLVAR, which also calculates similar weighted-averages from the $x$ and $y$ positions of the input atoms.

The ensemble average of the order parameter is calculated by computing the function inside the angle brackets in eq. (B.1) every step and by averaging over 25 trajectory snapshots. The final ensemble average that is calculated in this way is shown in fig. B1. This result is unsurprising: there is a region in the center of the box where the average value of $s_{i}$ is large and where the structure is a solid. In the remainder of the box, however, where the structure is liquid, the average value of $s_{i}$ is small.

It is easy to adjust the PLUMED input that was used to generate fig. B1 to calculate two or three dimensional profiles. Furthermore, by taking advantage of the STRIDE parameter in the DUMPGRID command and the CLEAR parameter in the MULTICOLVARDENS command we can easily adjust the number of frames over which we calculate the ensemble average. Finally, we can obviously change the quantity we are averaging by using some action besides FCCUBIC to calculate the symmetry functions that are used to calculate the average field in MULTICOLVARDENS.

\section{Appendix B.2. Finding contours}

As discussed in section 2 our aim is not to simply calculate the average value of some symmetry function. Instead we would like to calculate the surface that separates the liquid phase from the solid phase and to investigate how this surface fluctuates as the simulation progresses. Figure B2 shows that this objective is not unreasonable. The atoms in a single snapshot of the trajectory are shown colored according to their scaled $s_{i}$ parameter (see eq. (A.1)). The atoms with a high $s_{i}$ value that therefore have an environment that resembles that found in the solid are colored in blue while those 


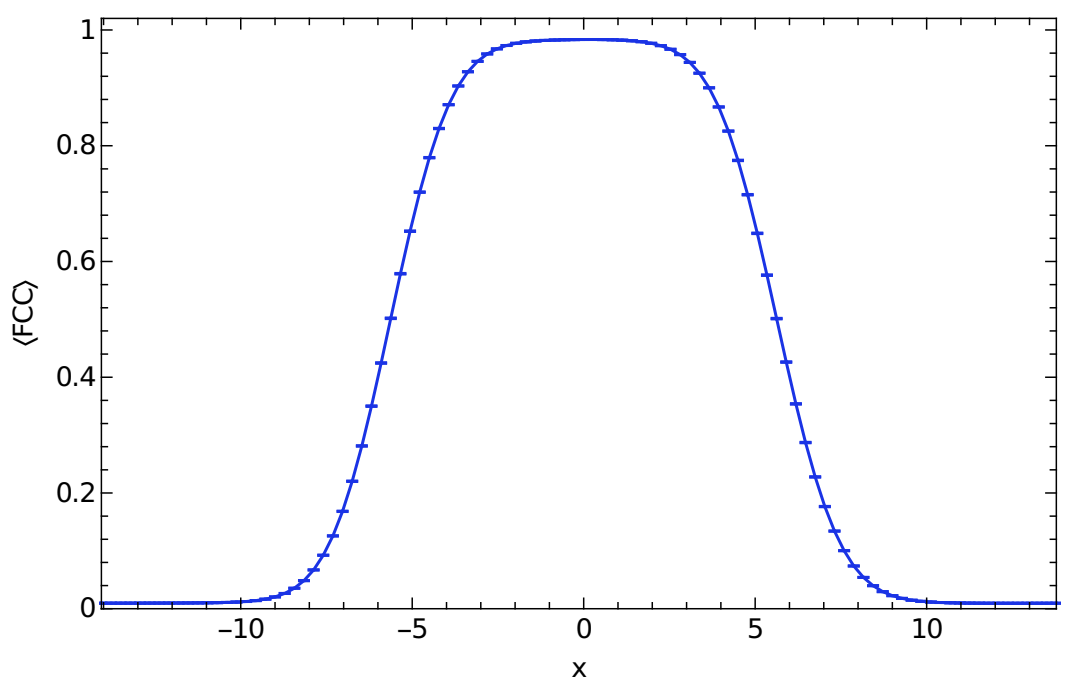

Figure B1. The average value of the atomic order parameter defined in equations A.1 and A.2 as a function of $z$. This profile was calculated using the method described in equation 2 and shows clearly that the atoms in the center of the box are arranged as they would be in a solid, while those at the edges of the box are arranged as they would be in a liquid.

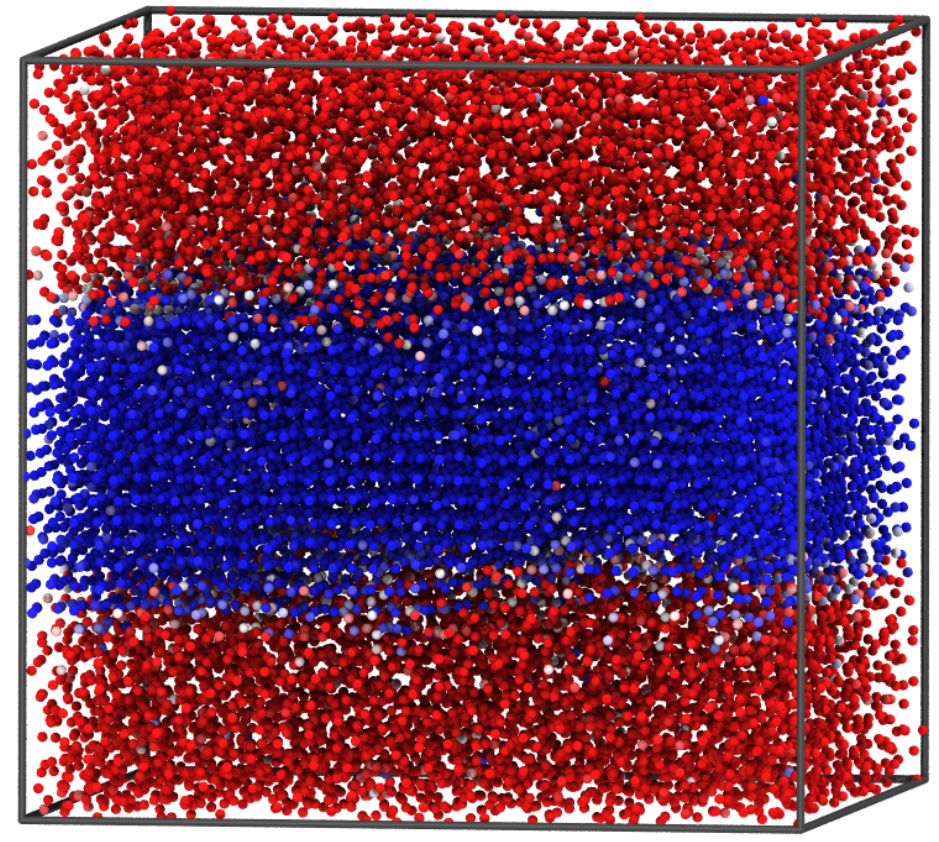

Figure B2. A snapshot from one of our molecular dynamics simulations of the interface between solid and liquid Lennard-Jones. In this figure the atoms are colored according to the value they have for the symmetry function defined in eq. (A.1) and A.2. Those colored blue have a high value for $s_{i}$ and are thus sat in an environment that resembles that found in the solid. Those colored in red have a low value for this quantity. The structure surrounding these atoms is thus more liquid like. 
with a low, liquid-like value are colored in red. This figure shows clearly that the box can be partitioned into liquid and solid parts and that there is clear, albeit non-flat, dividing surface between these regions. The following input to PLUMED uses our implementation of the Willard-Chandler surface to find the location of this dividing surface.

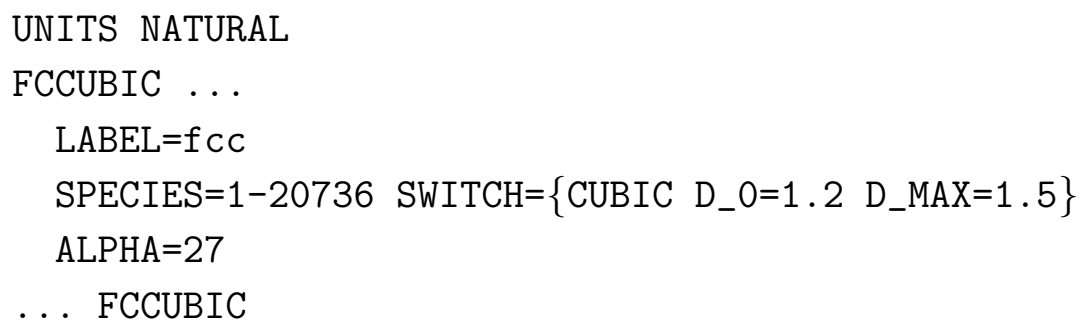

This input instructs PLUMED to calculate the scaled value of $s_{i}$ using equation A.1 for each of the atoms in the system. These quantities are then transformed using equation A.2 and a spatial average for the resulting quantity is evaluated on a three dimensional grid that covers the whole simulation cell using equation 2. The 2Dmanifold of points for which the resulting spatial average of the transformed and scaled $s_{i}$ values equals 0.5 is then found using a variant on the marching cubes algorithm. This algorithm searches over all the grid points at which the spatial average is evaluated. At each grid point the difference between the value of the spatial average at that point and the target value of 0.5 is evaluated. The sign of this quantity is then compared with the sign of the same quantity evaluated at the adjacent grid points in the $x, y$ and $z$ directions. If the sign of this quantity on adjacent grid points in any of these directions do not match then the manifold separating the solid from the liquid must pass through the vector connecting these two grid points. We thus find points on the manifold separating the solid from the liquid by searching between pairs of grid points with opposing signs for $\varphi(x, y, z)-\varphi_{0}$ using Brent's root-finding algorithm [39, 40] and splines that interpolate the value of the spatial average between grid points from the values that are evaluated at the grid points [39]. The positions of the resulting points 


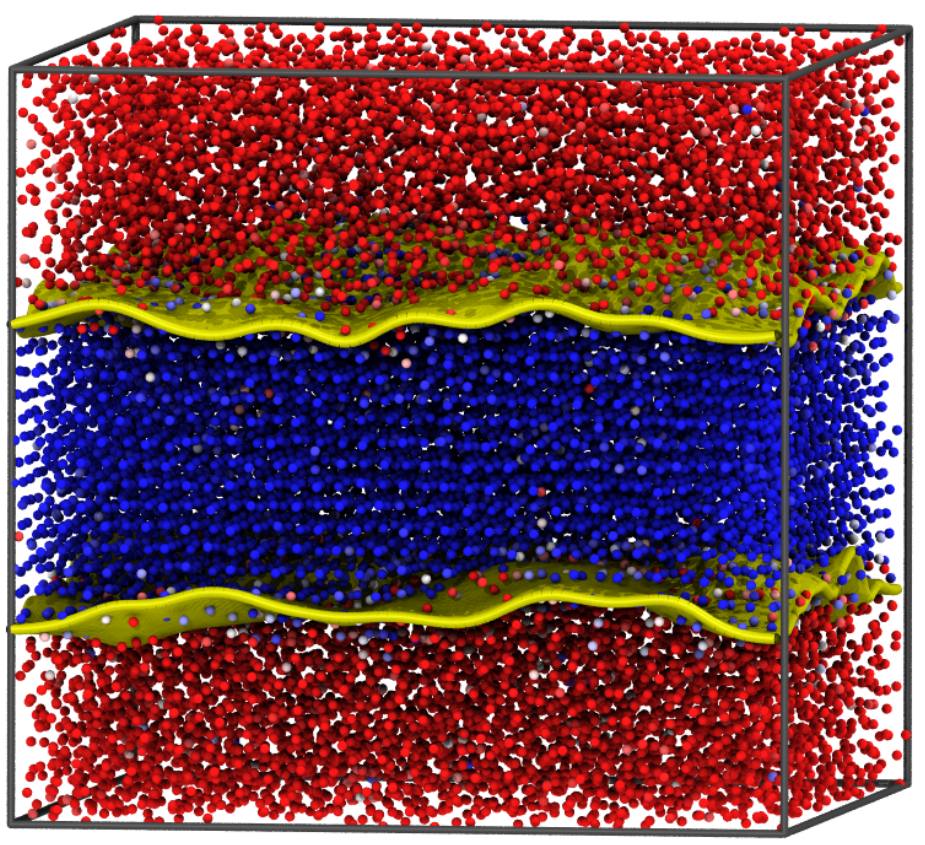

Figure B3. A snapshot from one of our molecular dynamics simulations of the interface between solid and liquid Lennard-Jones with the atoms colored as described in the caption to figure B2. The Willard-Chandler surface that separates the solid and liquid phases is shown in yellow in this figure.

are then output to a file. A surface passing through the set of points on the manifold that were found when we analyzed the configuration shown in figure B2 is shown in figure B3.

It is important to note that in the PLUMED input that we used for this calculation the CLEAR and STRIDE parameters in the MULTICOLVARDENS action are set equal. Setting these two parameters to be equal ensures that the grid accumulators are reset after each calculation of the contour. The contour found thus provides information on the instantaneous location of the interface between the solid and liquid parts of the cell. If the CLEAR parameter is set larger than the STRIDE parameter then an ensemble average for the spatial average of $s_{i}$ is computed over a number of trajectory frames. One can then use FIND_CONTOUR to find the location of the dividing surface for this averaged profile.

\section{Appendix B.3. Finding surfaces}

The marching cubes algorithm that was used to find points on the manifold separating the solid from the liquid in the previous section is useful as information on the expected geometry of the contour is not required by this algorithm. It can thus find the manifold separating the phases regardless of whether the phase has a slab geometry, a spherical geometry or even a toroidal geometry. Although this flexibility is useful in general, it is often unnecessary and can in fact make subsequent analysis more complicated. For this reason we provide a second method for finding the location of the dividing surface. 


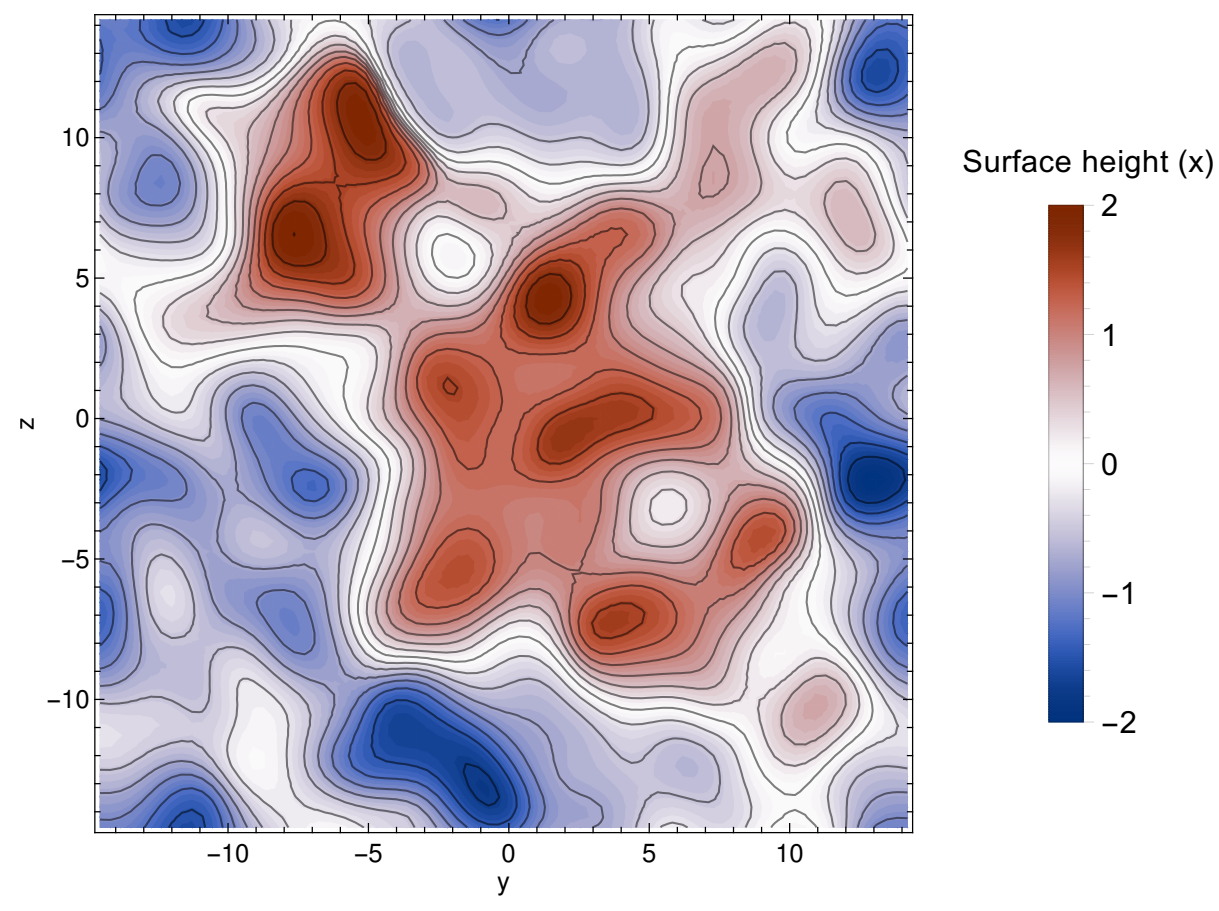

Figure B4. A contour plot showing the height of the interface between the solid and liquid phases of Lennard Jones versus $x$ and $y$ for the snapshot of the trajectory shown in figure B2.

In this second method we only search for points on the dividing surface in the direction perpendicular to the interface between the two phases. Furthermore, in this second method we do not calculate the value of the spatial average on a grid that encompasses the entirety of the simulation cell. We instead only calculate the value of the spatial average in the parts of the box that we suppose are close to one of the dividing surfaces. The advantage of changing the manner in which the location of the surface is found in these two ways is illustrated in figure B4. As this figure shows this new method finds one value for the height, $z$, of the dividing surface for each pair of $x$ and $y$ values in our three dimensional grid. We can thus use a contour plot to display $z(x, y)$ and we can interpolate and integrate this interfacial-height-profile function.

The PLUMED input that was used to generate the contour plot in figure B4 from the configuration shown in the left most panel is given below.

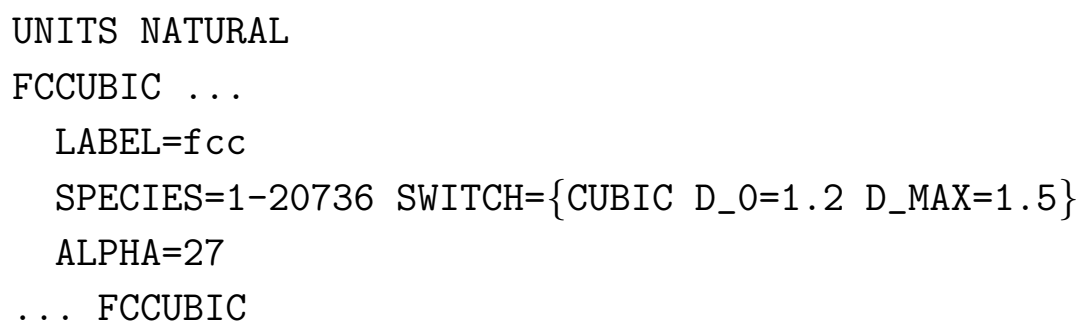


center: CENTER_OF_MULTICOLVAR DATA=smapfcc

MULTICOLVARDENS ...

LABEL $=$ dens

DATA $=$ smap $\mathrm{CC} \quad$ ORIGIN=center $\mathrm{DIR}=\mathrm{xyz}$

NBINS $=50,80,80$ BANDWIDTH $=1.0,1.0,1.0$

XREDUCED XLOWER $=0.0$ XUPPER $=20.0$

STRIDE $=1$ CLEAR $=1$

... MULTICOLVARDENS

FIND_CONTOUR_SURFACE . . .

LABEL= contour

GRID $=$ dens CONTOUR $=0.5$ SEARCHDIR $=x$

STRIDE $=1$ CLEAR $=1$

... FIND_CONTOUR_SURFACE

DUMPGRID GRID=contour FILE=contour . dat

The keyword CONTOUR is the same as for the action FIND_CONTOUR, while the keyword SEARCHDIR tells PLUMED in which directions to search for the dividing surface. It is worth noting that the output from the contour surface found by the FIND_CONTOUR_SURFACE action is stored as a function on a grid within PLUMED. In other words, the object output by this action has the same type as the object output by MULTICOLVARDENS. Everything we can do to the output from MULTICOLVARDENS - be that printing it to a file, interpolating the function, integrating it or even finding contours within it - can thus also be done to the output of FIND_CONTOUR_SURFACE.

In this work we took the output from FIND_CONTOUR_SURFACE and performed a discrete Fourier transform of $z(x, y)$ using the FFTW library [41]. To do this within PLUMED the following lines need to be added to the input above:

FOURIER_TRANSFORM $\ldots$

$\mathrm{LABEL}=\mathrm{ft}$

GRID=contour

STRIDE $=10$ CLEAR=25

FT_TYPE=norm FOURIER_PARAMETERS $=-1,1$

... FOURIER_TRANSFORM

DUMPGRID GRID $=f t$ FILE $=$ fourier . dat STRIDE $=10$

The keyword FT_TYPE here tells PLUMED the kind of data that is required in the Fourier transformed function that is output. The possible values control what operations 
are performed after the discrete Fourier transform is computed: "abs" tells PLUMED that only the complex moduli of the Fourier coefficients are required, while "norm" tells PLUMED that the norm of the complex modulus is required. The full, complex Fourier coefficients are only returned from FFTW to PLUMED when the keyword FT_TYPE is set to "complex" or left out. Furthermore, when this is done the user must think of these complex numbers on a grid as if they are vectors.

An additional keyword is implemented in the FOURIER_TRANSFORM action to control how the output should be normalized. The keyword FOURIER_PARAMETERS takes two numerical values $(a, b)$ that define the normalization and the type (forward or backward) of the Fourier transform that is to be applied to the data. The role these parameters play in the operation can be understood by considering the following $1 \mathrm{D}$ example, in which an array $X$ of size $n$ is transformed:

$$
Y_{k}=\frac{1}{n^{(1-a) / 2}} \sum_{j=0}^{n-1} X_{j} \exp (2 \pi i b j k / n) .
$$

Notice once again that the output from the FOURIER_TRANSFORM command is a function evaluated at points on a grid and that within PLUMED the output from this action can thus be treated as such. Further note that in the example above the CLEAR parameter in the line defining the Fourier transform is set much larger than the STRIDE parameter. This command is thus calculating an ensemble average for the Fourier transform. In other words, the location of the dividing surface is updated based on the positions of the atoms in each of the trajectory frames and Fourier transformed. The above command thus calculates the ensemble average of this Fourier transform over multiple trajectory frames.

\section{References}

[1] W. Kurz and D. J. Fisher. Fundamental of Solidification. Trans Tech Publications, 1998.

[2] J.A. Dantzig and M. Rappaz. Solidification. CRC Press (EPFL Press), 2009. Chap. 7.

[3] Y. Nagata et al. "Nuclear Quantum Effects Affect Bond Orientation of Water at the Water-Vapor Interface". In: Phys. Rev. Lett. 109 (2012), p. 226101.

[4] J. Liu et al. "A Surface-Specific Isotope Effect in Mixtures of Light and Heavy Water". In: J. Phys. Chem. C 117 (2013), pp. 2944-2951.

[5] Y. R. Shen. "Surface properties probed by second-harmonic and sum-frequency generation". In: Nature 337.6207 (Feb. 1989), pp. 519-525.

[6] B. Cheng and M. Ceriotti. "Bridging the gap between atomistic and macroscopic models of homogeneous nucleation". In: The Journal of Chemical Physics 146.3 (Jan. 2017), p. 034106. 
[7] J.J Hoyt, M. Asta, and A. Karma. "Method for computing the anisotropy of the solid-liquid interfacial free energy". In: Physical Review Letters 86.24 (2001), p. 5530 .

[8] R. L. Davidchack and B. B Laird. "Direct calculation of the hard-sphere crystal/melt interfacial free energy". In: Physical review letters 85.22 (2000), p. 4751.

[9] R. L. Davidchack and B. B Laird. "Direct calculation of the crystal-melt interfacial free energies for continuous potentials: Application to the Lennard-Jones system". In: The Journal of chemical physics 118.16 (2003), pp. 7651-7657.

[10] S. Angioletti-Uberti et al. "Solid-liquid interface free energy through simulations". In: Phys. Rev. B 81.12 (2010), p. 125416.

[11] U. R. Pedersen et al. "Computing Gibbs free energy differences by interface pinning". In: Physical Review B 88.9 (2013), p. 094101.

[12] B. Cheng, G. A. Tribello, and M. Ceriotti. "Solid-liquid interfacial free energy out of equilibrium". In: Phys. Rev. B 92.18 (2015), p. 180102.

[13] R.E. Rozas and J. Horbach. "Capillary wave analysis of rough solid-liquid interfaces in nickel". In: Europhys. Lett. 93 (2011), p. 26006.

[14] R. L. Davidchack, J. R. Morris, and B. B. Laird. "The anisotropic hard-sphere crystal-melt interfacial free energy from fluctuations". In: The Journal of Chemical Physics 125.9 (2006), p. 094710.

[15] J. Benet, L. G. MacDowell, and E. Sanz. "Interfacial free energy of the $\mathrm{NaCl}$ crystal-melt interface from capillary wave fluctuations". In: The Journal of Chemical Physics 142.13 (2015), p. 134706.

[16] M. Ambler et al. "Solid-liquid interfacial free energy of ice Ih, ice Ic, and ice 0 within a mono-atomic model of water via the capillary wave method". In: The Journal of Chemical Physics 146.7 (2017), p. 074701.

[17] A. P. Willard and D. Chandler. "Instantaneous Liquid Interfaces". In: The Journal of Physical Chemistry B 114.5 (2010), pp. 1954-1958.

[18] M. Sega et al. "How Is the Surface Tension of Various Liquids Distributed along the Interface Normal?" In: The Journal of Physical Chemistry C 120.48 (2016), pp. 27468-27477.

[19] M. Jorge et al. "A Critical Assessment of Methods for the Intrinsic Analysis of Liquid Interfaces: 2. Density Profiles". In: The Journal of Physical Chemistry C 114.43 (2010), pp. 18656-18663.

[20] D. T. Allen and C. D. Lorenz. "A novel method for constructing continuous intrinsic surfaces of nanoparticles". In: Journal of Molecular Modeling 23.8 (July 2017), p. 219.

[21] G. A. Tribello et al. "PLUMED 2: New feathers for an old bird". In: Computer Physics Communications 185.2 (2014), pp. 604-613. 
[22] F. Giberti et al. "Insight Into The Nucleation Of Urea Crystals From The Melt". In: Chem. Eng. Sci. 2013 Danckwerts Special Issue on Molecular Modelling in Chemical Engineering 121 (Jan. 2015), pp. 51-59.

[23] G. A. Tribello et al. "Analyzing and Driving Cluster Formation in Atomistic Simulations". In: Journal of Chemical Theory and Computation 13.3 (2017), pp. $1317-1327$.

[24] P. J. Steinhardt, D. R. Nelson, and M. Ronchetti. "Bond-orientational order in liquids and glasses". In: Physical Review B 28.2 (July 1983), pp. 784-805. (Visited on $03 / 16 / 2016)$.

[25] W. Lechner and C. Dellago. "Accurate determination of crystal structures based on averaged local bond order parameters". In: The Journal of Chemical Physics 129.11 (Sept. 2008), p. 114707.

[26] S. Auer and D. Frenkel. "Prediction of crystal-nucleation rates for spherical colloids: a computational approach". In: Ann. Rev. Phys. Chem. 55 (2004), pp. 333-361. (Visited on 11/28/2014).

[27] J. Hoyt, M. Asta, and A. Karma. "Method for Computing the Anisotropy of the Solid-Liquid Interfacial Free Energy". In: Phys. Rev. Lett. 86 (2001), pp. 55305533.

[28] J. R. Morris and X. Song. "The anisotropic free energy of the Lennard-Jones crystal-melt interface". In: J. Chem. Phys. 119 (2003), pp. 3920-3925. (Visited on 02/18/2015).

[29] C. A. Becker et al. "Atomistic simulations of crystal-melt interfaces in a model binary alloy: Interfacial free energies, adsorption coefficients, and excess entropy". In: Phys. Rev. B 79.5 (2009), p. 054109. ISSN: 1098-0121, 1550-235X.

[30] D. Du, H. Zhang, and D. J. Srolovitz. "Properties and determination of the interface stiffness". In: Acta. Mater. 55 (2007), pp. 467-471.

[31] W.R. Fehlner and S.H. Vosko. "A product representation for cubic harmonics and special directions for the determination of the Fermi surface and related properties". In: Can. J. Phys. 54 (1976), p. 2159.

[32] B. Cheng, G. A. Tribello, and M. Ceriotti. "Solid-liquid interfacial free energy out of equilibrium". In: Phys. Rev. B 92 (18 Nov. 2015), p. 180102.

[33] S. Angioletti-Uberti. "The solid-liquid interface free-energy of $\mathrm{Pb}$ : comparison of theory and experiments". In: J. Phys. Cond. Matt. 23 (2011), p. 435008.

[34] M. Ceriotti, G. Bussi, and M. Parrinello. "Colored-Noise Thermostats à la Carte". In: J. Chem. Theory Comput. 6 (2010), pp. 1170-1180.

[35] H. Flyvbjerg and H.G. Petersen. "Error estimates on averages of correlated data". In: J. Chem. Phys. 91 (1989), pp. 461-466.

[36] D. Frenkel and B. Smit. Understanding Molecular Simulation. Second. London: Academic Press, 2002. 
[37] A. Laio and M. Parrinello. "Escaping free-energy minima". In: Proceedings of the National Academy of Sciences 99.20 (2002), pp. 12562-12566.

[38] B. Cheng, G. A. Tribello, and M. Ceriotti. The Gibbs free energy of homogeneous nucleation: from atomistic nuclei to the planar limit. Mar. 2017. arXiv: 1703. 06062 [cond-mat.stat-mech].

[39] W. H. Press et al. Numerical Recipes in $C++$. Cambridge, 2002.

[40] R. Brent. Algorithms for minimization without derivatives. Prentice-Hall, 1973. Chap. 5.

[41] M. Frigo and S. G. Johnson. "The Design and Implementation of FFTW3". In: Proceedings of the IEEE 93.2 (2005). Special issue on "Program Generation, Optimization, and Platform Adaptation", pp. 216-231. 